ARTICLE

DOI: $10.1038 / s 41467-018-04233-5$

\title{
Ambipolar ferromagnetism by electrostatic doping of a manganite
}

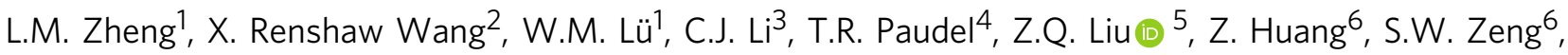 \\ Kun Han ${ }^{6}$, Z.H. Chen ${ }^{7,8}, 9$, X.P. Qiu (10 ${ }^{10}$, M.S. Li ${ }^{3}$, Shize Yang (1) ${ }^{11}$, B. Yang ${ }^{1}$, Matthew F. Chisholm ${ }^{11}$, \\ L.W. Martin (1) 7,8, S.J. Pennycook ${ }^{3}$, E.Y. Tsymbal ${ }^{4}$, J.M.D. Coey (i] ${ }^{12,13}$ \& W.W. Cao ${ }^{1,14}$
}

Complex-oxide materials exhibit physical properties that involve the interplay of charge and spin degrees of freedom. However, an ambipolar oxide that is able to exhibit both electron-doped and hole-doped ferromagnetism in the same material has proved elusive. Here we report ambipolar ferromagnetism in $\mathrm{LaMnO}_{3}$, with electron-hole asymmetry of the ferromagnetic order. Starting from an undoped atomically thin $\mathrm{LaMnO}_{3}$ film, we electrostatically dope the material with electrons or holes according to the polarity of a voltage applied across an ionic liquid gate. Magnetotransport characterization reveals that an increase of either electron-doping or hole-doping induced ferromagnetic order in this antiferromagnetic compound, and leads to an insulator-to-metal transition with colossal magnetoresistance showing electron-hole asymmetry. These findings are supported by density functional theory calculations, showing that strengthening of the inter-plane ferromagnetic exchange interaction is the origin of the ambipolar ferromagnetism. The result raises the prospect of exploiting ambipolar magnetic functionality in strongly correlated electron systems.

\footnotetext{
${ }^{1}$ Condensed Matter Science and Technology Institute, School of Science, Harbin Institute of Technology, Harbin 150081, China. ${ }^{2}$ School of Physical and Mathematical Sciences \& School of Electrical and Electronic Engineering, Nanyang Technological University, Singapore 639798, Singapore. ${ }^{3}$ Department of Materials Science and Engineering, National University of Singapore, Singapore 117575, Singapore. ${ }^{4}$ Department of Physics and Astronomy \& Nebraska Center for Materials and Nanoscience, University of Nebraska, Lincoln, Nebraska 68588, USA. ${ }^{5}$ School of Materials Science and Engineering, Beihang University, Beijing 100191, China. ${ }^{6}$ NUSNNI-NanoCore, National University of Singapore, Singapore 117411, Singapore. ${ }^{7}$ Department of Materials Science and Engineering, University of California, Berkeley, Berkeley, CA 94720, USA. ${ }^{8}$ Materials Sciences Division, Lawrence Berkeley National Laboratory, Berkeley, CA 94720, USA. ${ }^{9}$ School of Materials Science and Engineering, Harbin Institute of Technology, Shenzhen, Guangzhou 518055, China. ${ }^{10}$ Shanghai Key Laboratory of Special Artificial Microstructure Materials and Technology \& Pohl Institute of Solid State Physics \& School of Physics Science and Engineering, Tongji University, Shanghai 200092, China. ${ }^{11}$ Materials Science and Technology Division, Oak Ridge National Laboratory, Oak Ridge, TN 37831, USA.

${ }^{12}$ School of Physics, Trinity College, Dublin 2, Ireland. ${ }^{13}$ Faculty of Materials Science and Engineering, Beihang University, Beijing 100191, China.

${ }^{14}$ Department of Mathematics and Materials Research Institute, The Pennsylvania State University, University Park, PA 16802, USA. Correspondence and requests for materials should be addressed to X.R.W. (email: renshaw@ntu.edu.sg) or to W.M.Lü. (email: weiminglv@hit.edu.cn)
} 
M anipulating carriers of either sign and flipping the orientation of ferromagnetically coupled spins are operations that are basic to current information technology. An ambipolar ferromagnet that would allow the ordered spins to coexist with either electrons or holes in a single material, without modifying its crystal structure or chemical composition, is an appealing prospect. It could lead to advances in fundamental understanding of magnetic semiconductors and form a building block for new electric-field controlled spintronics based on the ability to create, control and detect the spin-polarized holes or electrons. Operating locally and reversibly, the electric-field offers notable advantages over magnetic field or hard chemical doping in the fabrication and operation of spintronic devices. Recently, there has been a lot of interest in ambipolar functionality, such as ambipolar conductivity in graphene ${ }^{1}$, black phosphorus ${ }^{2}$, and organic materials ${ }^{3}$. However, ferromagnetic semiconductors have so far been based on either electron-doped or hole-doping, but never both in the one material, precluding possible ambipolar spintronic devices, such as a dual-channel field-effect spin-filter or a field-effect spin valve.

Researchers are exploring various ways to create amipolar functionality in materials. Electrostatic doping ${ }^{4,5}$ of the mixedvalence manganites ${ }^{6}$ using an ionic liquid gate is a promising approach to try to realize ambipolar ferromagnetism, in light of the manganites' rich magnetic phase diagrams and the possibility of generating high carrier densities of up to $\sim 10^{15} \mathrm{~cm}^{-2}$, using an ionic liquid as gate dielectric. Ionic liquids have been used to achieve various other effects, including ambipolar doping layered materials $^{2}$, superconductivity $y^{4,7-14}$, charge density waves $^{15}$, metal-insulator transitions, ${ }^{16,17}$ and large resistive responses in mixed-valence manganites ${ }^{18-21}$. However, ambipolar ferromagnetism has proved difficult to achieve, owing to the challenge of varying the carrier density over a wide range while controlling the carrier-spin interactions. Nonetheless, from a material's point of view, as an end member of several mixed-valence manganite solid solutions, $\mathrm{LaMnO}_{3}$ (LMO) is a good candidate for the realization of ambipolar ferromagnetism. The manganese is trivalent $\left(\mathrm{Mn}^{3+} ; 3 d^{4}\right)$ in bulk LMO, which exhibits an A-type antiferromagnetic ground state, but offers possibilities for hosting ferromagnetism with both electron $\left(\mathrm{Mn}^{2+} ; 3 d^{5}\right)$ and hole $\left(\mathrm{Mn}^{4+}\right.$; $\left.3 d^{3}\right)$ doping 22,23 .

Here we have managed to achieve ambipolar ferromagnetism by electrostatically gating atomically thin LMO films using an ionic liquid as a gate dielectric.

\section{Results}

Basic characterization. Figure 1a shows the electric double-layer transistor (EDLT) with a planar device configuration fabricated on a three unit cell (uc) thick LMO film ${ }^{24}$ grown on $\mathrm{SrTiO}_{3}(001)$ (STO) with an ionic liquid as the electrolyte (Methods section). Figure $1 \mathrm{~b}$ illustrates the atomically flat surface of LMO after the gating measurements, revealed by an atomic force microscope. The high quality surface after gating indicates that etching of the
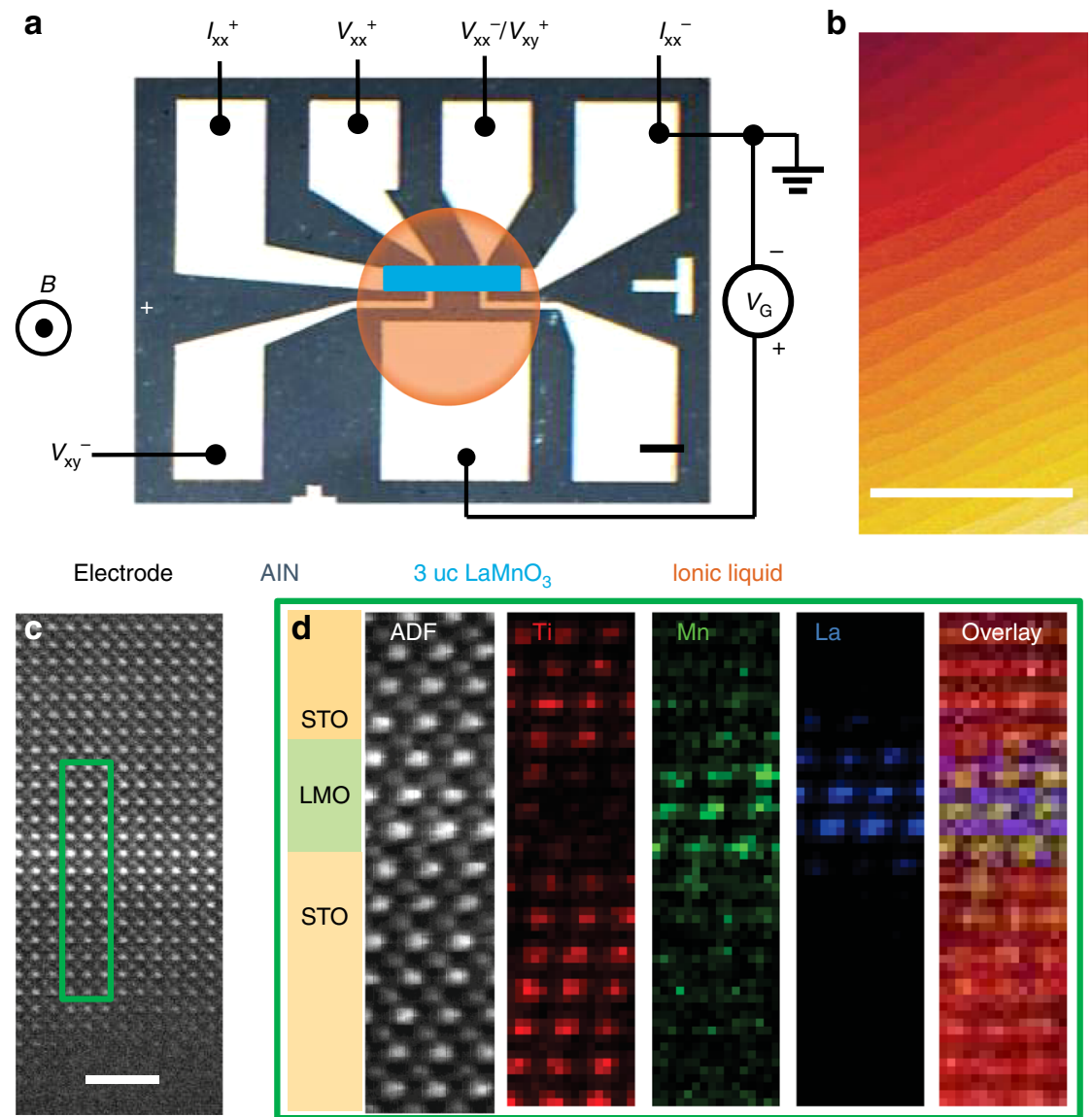

Fig. 1 Schematic and structural characterization of the electric double-layer transistor. a Schematic of the device, fabricated by ultraviolet lithography. The exposed area of $\mathrm{LaMnO}_{3}$ is $300 \mu \mathrm{m}$ in length and $50 \mu \mathrm{m}$ in width. The scale bar is $100 \mu \mathrm{m}$. b Atomically flat surface of 3 unit cell (uc) LaMnO film grown on a $\mathrm{SrTiO}_{3}$ (001) substrate characterized by atomic force microscopy. The scale bar is $1 \mu \mathrm{m}$. c High-angle annular dark field (HAADF) image of the $\mathrm{SrTiO}_{3}-$ capped sample. The scale bar is $2 \mathrm{~nm}$. $\mathbf{d}$ EELS of the $\mathrm{SrTiO}_{3}$-capped sample. The corresponding EELS region is indicated in the green box in the HAADF image (c) 
material due to ionic liquid gating is negligible. In addition, we performed high-resolution cross-section scanning transmission electron microscopy (STEM) and electron energy loss spectroscopy (EELS) on a 3 uc LMO sample with an overlayer of STO. The STO overlayer was added in order to avoid damage during the STEM sample preparation to correctly characterize the interface between LMO and STO (see Supplementary Note 7). Figure 1c shows a high-angle annular dark field (HAADF) image of the sample along the [010] zone axis. Figure 1d shows the EELS results of the region of green square in Fig. 1c, demonstrating that the extent of intermixing at the LMO-STO interface is limited to $1 \mathrm{uc}$. The ultrathin layer thickness is a prerequisite for our study, because the required modulation of the carrier density is very large. In thick films, the induced carriers will be spread out, resulting in little modulation of the density. Furthermore, the surface and interface states in ultrathin films are more sensitive to the external modulations, such as electrostatic doping ${ }^{25,26}$. Carrier doping was realized by applying a gate voltage $\left(V_{\mathrm{G}}\right)$ to the double-layer capacitor formed by the ionic liquid. The sheet resistance $\left(R_{\mathrm{S}}\right)$ of the LMO film at $300 \mathrm{~K}$ decreases linearly on both sides of zero-voltage, as shown in Fig. 2a. This gating response is typical of an ambipolar material. It is important to note that no hysteresis is observed while sweeping $V_{\mathrm{G}}$, demonstrating that diffusion of oxygen vacancies is negligible during the gating experiments.

Electrostatic gating. In order to further investigate the carrier behavior and the intrinsic magnetic properties of the material, the Hall resistivity $\left(\rho_{x y}\right)$ of the 3 uc LMO film was measured at
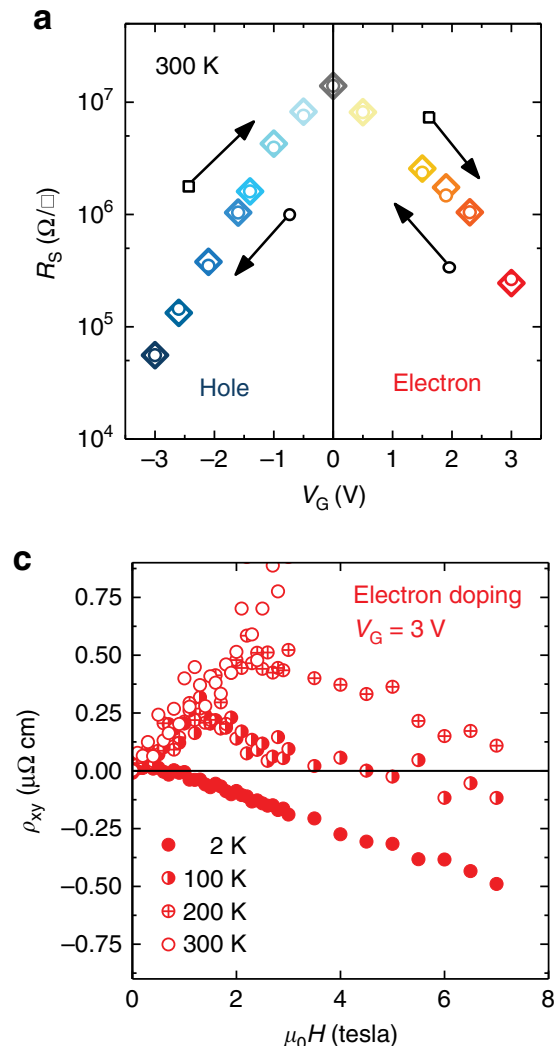

different temperatures for $V_{\mathrm{G}}= \pm 3 \mathrm{~V}$. The Hall effect in a ferromagnet is a combination of the ordinary Hall effect (OHE) and the anomalous Hall effect $(\mathrm{AHE})^{27,28}$. In ferromagnetic mixedvalence manganites, the $\mathrm{OHE}$ and $\mathrm{AHE}$ contributions to $\rho_{x y}$ have opposite trends, resulting in an anomalous $\rho_{x y}$ behavior different from that in ordinary ferromagnetic materials. The AHE starts to dominate above $\sim 100 \mathrm{~K}$, increases sharply around the Curie temperature $\left(T_{\mathrm{C}}\right)$, peaking at a temperature roughly $30 \mathrm{~K}$ above $T_{C}$, and decreases slowly at higher temperatures ${ }^{29}$. Figure $2 \mathrm{~b}, \mathrm{c}$ shows $\rho_{x y}$ of LMO at various temperatures in the electron-doped and hole-doped regimes, exhibiting an AHE which is in good agreement with studies on $\mathrm{La}_{1-x} \mathrm{~A}_{x} \mathrm{MnO}_{3}$ $(\mathrm{A}=\mathrm{Ba}, \mathrm{Ca}, \mathrm{Pb}, \mathrm{Ce}$, and $\mathrm{Sr})$ single crystals ${ }^{23,30,31}$. Typically, the AHE in hole-doped manganites, such as $\mathrm{La}_{1-x} \mathrm{Sr}_{x} \mathrm{MnO}_{3}$ and $\mathrm{La}_{1}$ ${ }_{-x} \mathrm{Ca}_{x} \mathrm{MnO}_{3}$, is prominent ${ }^{31}$ at temperatures close to the Curie temperature, $T_{C}$, namely in the higher temperature region of the metallic phase, where $\mathrm{d} \rho_{x x} / \mathrm{d} T$ is positive and the lower temperature region of the insulating phase, where $\mathrm{d} \rho_{x x} / \mathrm{d} T$ is negative. When the temperature is well below or above the $T_{C}$, the Hall resistance becomes linear in magnetic field. These generic features are consistent with our experimental observation. The maximum magnitude of the AHE for $\mathrm{La}_{1-x} \mathrm{Sr}_{x} \mathrm{MnO}_{3}$ and $\mathrm{La}_{1-x} \mathrm{Ca}_{x} \mathrm{MnO}_{3}$ are $\sim 0.65$ and $1.3 \mu \Omega \mathrm{cm}$, which is also consistent with the magnitude of the AHE in our study. Since the AHE is due to broken time-reversal symmetry, typically as a result of spin-orbit coupling in a ferromagnetic phase ${ }^{28,32,33}$, the data demonstrate the ferromagnetic nature of the films under both positive and negative gating.

The carrier densities of the electrons and holes under different gate voltages were calculated from the Hall measurement at $2 \mathrm{~K}$,
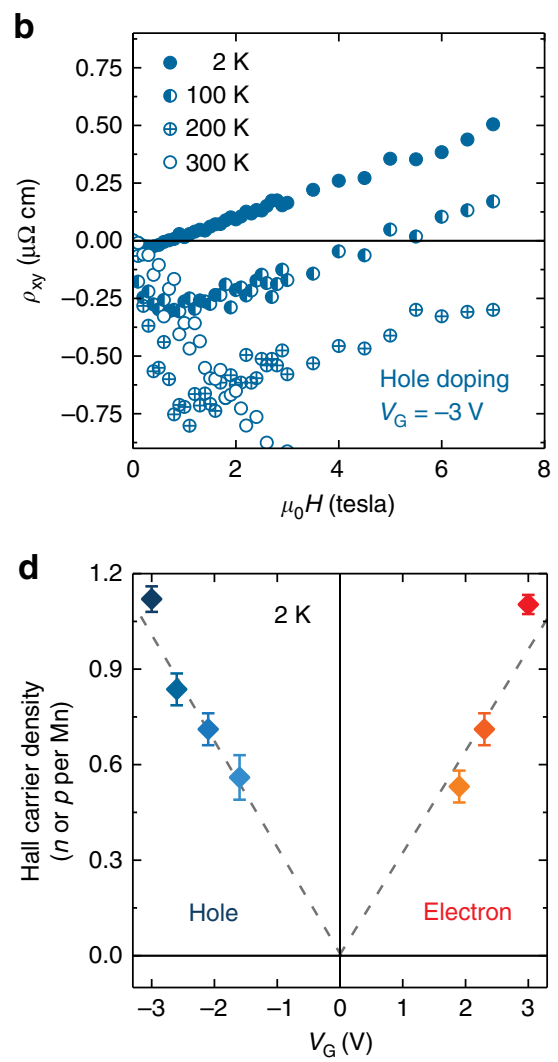

Fig. 2 Transport properties of $3 \mathrm{uc} \mathrm{LaMnO}_{3}$ film at various gate voltages $\left(V_{\mathrm{G}}\right)$ and temperatures. a The sheet resistance as a function of $V_{\mathrm{G}}$ at $300 \mathrm{~K}$. No loop is seen, indicating that effects of oxygen migration are negligible. $\mathbf{b}, \mathbf{c}$ Hall resistivity $\left(\rho_{x y}\right)$ of $\mathrm{LaMnO}_{3}$ under of $\mathrm{V}_{\mathrm{G}}=-3 \mathrm{~V}(\mathbf{b})$ and $3 \mathrm{~V}(\mathbf{c})$ at different temperatures. temperatures. Due to the presence of high noise levels in $\rho_{x y}(300 \mathrm{~K})$ for magnetic field above 3 Tesla, the high-field data of $\rho_{x y}$ at $300 \mathrm{~K}$ are omitted. $\mathbf{d}$ Hall carrier density expressed in electron or hole content per $\mathrm{Mn}$ site based on the Hall results at $2 \mathrm{~K}$ and various $V_{\mathrm{G}}$. The error bars at these $V_{\mathrm{G}}$ were calculated based on the fluctuation of $\rho_{x y}$ within the ordinary Hall effect $(\mathrm{OHE})$ region at $2 \mathrm{~K}$ 
and are shown in Fig. $2 \mathrm{~d}$ with $n(p)$ representing the electron(hole) content per $\mathrm{Mn}$. At $2 \mathrm{~K}, \rho_{x y}$ exhibits a linear variation with magnetic field $\left(\mu_{0} H\right)$, and opposite signs of the slope are observed according to the sign of $V_{\mathrm{G}}$, revealing electron and hole characteristics of the LMO under positive and negative gate voltages, respectively. However, the values of carrier density deduced at $2 \mathrm{~K}$ are generally much larger than the nominal doping concentration ${ }^{23,28,30,31}$, although the sign of the slope of $\rho_{x y}$ at low temperature is correct in our study, where the Hall carrier density of doped LMO achieved at $\pm 3 \mathrm{~V}$ corresponds to roughly one electron or hole per $\mathrm{Mn}$ site. The abnormally large Hall carrier density of manganites is well documented in the existing literature $23,28,30,31$, but its mechanism remains unexplained. For instance, nominal chemically doped $\mathrm{La}_{1-x} A_{x} \mathrm{MnO}_{3}$ thin films with $\sim 0.1$ e per $\mathrm{Mn}$ always exhibit roughly one, or even more, electrons or holes per Mn site in Hall measurements at $\sim 2$ $\mathrm{K}$. Nevertheless, a linear modulation of carrier density per $\mathrm{Mn}$ is confirmed. As shown in Fig. 2d, the Hall carrier density, which was estimated based on the normal Hall resistance regime of $\rho_{x y}$, shows that the gating effect on carriers is roughly symmetric on both sides. Although the doping effect is often found to be asymmetric due to the difference in the size of the positive and negative ions in the liquid, the capacitance for electron-doped and hole-doping can also be symmetric in some cases ${ }^{34,35}$.

Ambipolar ferromagnetism. The realization of ambipolar ferromagnetism is further confirmed by the variation of studies of sheet resistance $R_{\mathrm{S}}$ as a function of temperature for various gate voltages. Figure $3 \mathrm{a}$, b show the $R_{\mathrm{S}}$ vs. temperature curves of the 3 uc LMO under positive or negative $V_{\mathrm{G}}$. By increasing the negative $V_{\mathrm{G}}$ (hole accumulation, Fig. $3 \mathrm{a}$ ), the $R_{\mathrm{S}}$ vs temperature curves
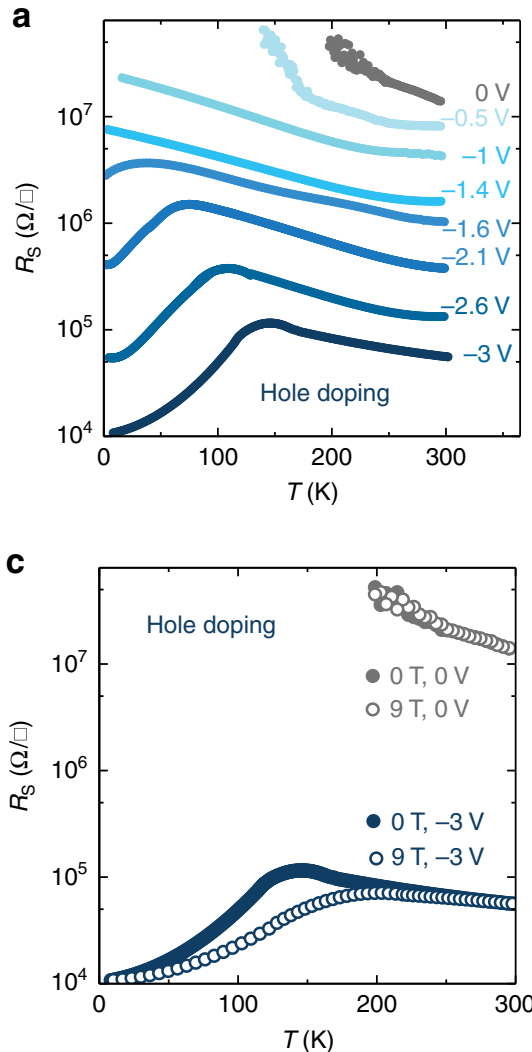

exhibit a metal-insulator transition (MIT) with a resistivity peak at a temperature $T_{\mathrm{P}}$, accompanying the decrease of resistivity. The same trend is observed when $V_{\mathrm{G}}$ is positive (electron accumulation, Fig. $3 \mathrm{~b}$ ). Below $T_{\mathrm{P}}$, electron-doped and hole-doped LMO both exhibit metallic behavior. The sheet resistance of our samples are quantitatively consistent with the resistance quantum $(h /$ $e^{2}=25.8 \mathrm{k} \Omega$ ), and the well-documented MIT in manganite. In the temperature regime above the MIT, the system is nonmagnetic and enters a semiconducting state. This is consistent with all understood cases with a resistance greater than $h / e^{2}$. In the temperature regime near and below the MIT, the resistivity of our sample is quantitatively consistent with the well-documented resistivity of manganite materials. For example, the resistivity of our samples at the peak temperature is $\sim 10^{-1}$ to $10^{-2} \Omega \mathrm{cm}$, showing the same value as the reported resistivity at the peak temperature $T_{\mathrm{P}}{ }^{22,36}$. Furthermore, $T_{\mathrm{P}}$ in both electron-doped and hole-doped regimes increases upon applying a 9 Tesla magnetic field (Fig. 3c, d), and colossal magnetoresistance (CMR) is observed. The field-induced increase of $T_{\mathrm{P}}$ resembles that of all other chemically doped ferromagnetic manganites, proving that the ferromagnetic metallic state induced by both positive and negative gating lies within LMO films, rather than at the surface of the STO substrate ${ }^{37}$

Electron-hole asymmetry. Strikingly, the doping dependence of $T_{\mathrm{P}}$ for electron doping is less sensitive than that for hole doping. Figure $4 \mathrm{a}$ presents a contour plot of normalized sheet resistance $\left(R_{\mathrm{S}}(T) / R_{T \mathrm{p}}\right)$ vs temperature and $V_{\mathrm{G}}$. The contour plot shows that $T_{\mathrm{P}}$ increases asymmetrically for positive and negative gating. Figure $4 \mathrm{~b}$ summarizes the data on $T_{\mathrm{P}}$ as a function of $V_{\mathrm{G}}$, demonstrating the electron-hole asymmetry of the CMR, and of
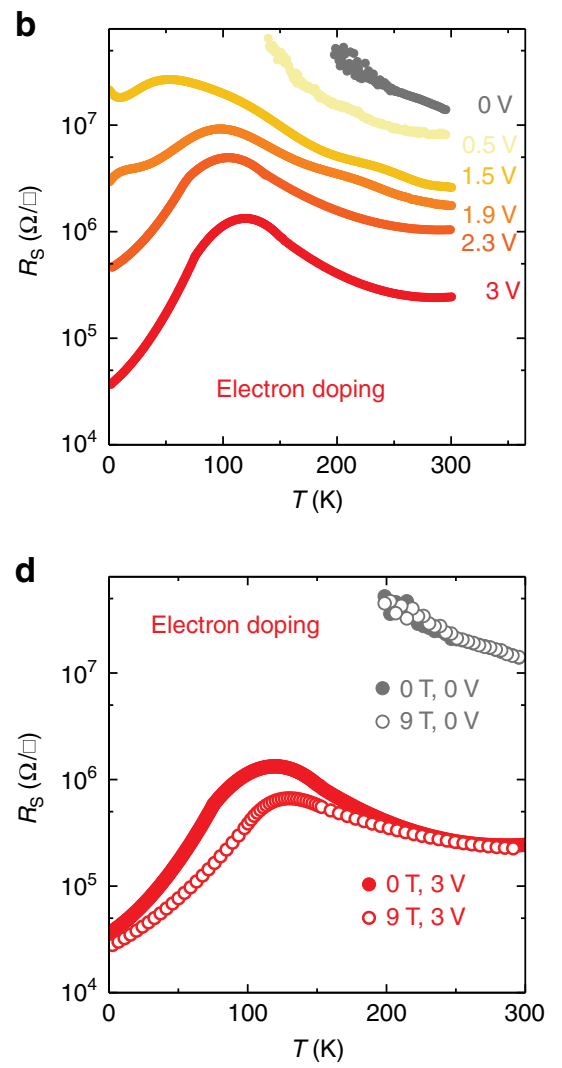

Fig. 3 Metal-insulator transition (MIT) in ionic liquid-gated $3 \mathrm{uc} \mathrm{LaMnO}_{3}$. a, b The sheet resistance as a function of gate voltages. Both negative (a) and positive (b) voltage regimes show a MIT, accompanying the reduction of sheet resistance. $\mathbf{c}$, $\mathbf{d}$ Temperature dependence of magnetoresistance in the holedoped and electron-doped 3 uc $\mathrm{LaMnO}_{3}$ film 
the ferromagnetic ordering temperature. The electron-hole asymmetry is further confirmed by the asymmetric magnitude and characteristic temperature of the temperature-dependent magnetoresistance (MR), defined as $(R(H)-R(0)) / R(0)(\%)$, for electron-doped and hole-doped LMO film (Fig. 4c).

Density functional theory calculations. First-principles density function theory (DFT) calculations were employed to understand the origin of the asymmetric ambipolar ferromagnetism (see Supplementary Note 6 for details). The magnetism in LMO can be described by an isotropic exchange Hamiltonian, $H=-\sum_{<i j>} J_{i j} S_{i} \cdot S_{j}$, where $J_{i j}$ is the exchange interaction between neighboring $\mathrm{Mn}$ ions with localized spin moments $S_{i}$ and $S_{j}$. The intra-plane and inter-plane exchange interactions have different values. The interplay of intra-plane $J_{a b}$ and inter-plane $J_{c}$ determines overall magnetic ordering (Supplementary Note 6). In fully relaxed bulk LMO, the intra-plane exchange $J_{a b}$ is ferromagnetic, whereas the inter-plane exchange $J_{c}$ is antiferromagnetic, which leads to the A-type antiferromagnetic ordering ${ }^{38}$. In the biaxially strained epitaxial films, we find that both exchange interactions are ferromagnetic, which results in overall ferromagnetic ordering. In the mean-field approximation, the strength of ferromagnetism is determined by the sum of the exchange parameters, $J=J_{c}+J_{a b}$. We find that $J$ increases with both electron and hole doping (Fig. 4d), which is largely due to the increasing ferromagnetic inter-plane exchange $J_{c}$ (see Supplementary Figure $6 \mathrm{~g}$ ). The asymmetry between electron and hole doping is caused by the intra-plane exchange $J_{a b}$, which increases with hole doping but decreases with electron doping (see Supplementary Figure $6 \mathrm{~g}$ ). The results for the exchange coupling $J$ (Fig. 4d) indicate that the enhancement of ferromagnetism is more sensitive to the hole doping than to the electron doping, which is consistent with the experimental results for the ferromagnetic ordering temperature (Fig. 4b).

The effect of doping on the exchange parameters can be traced to the change in orbital overlap due to the Jahn-Teller distortions ${ }^{39}$. The Jahn-Teller effect leads $\mathrm{Mn}^{3+}$ ions bonded to oxygen to have one longer bond, $b_{1}$, and one shorter bond, $b_{s}$. The ratio of $b_{1} / b_{\mathrm{s}}$ represents the extent of the distortion. As shown in Fig. $4 \mathrm{~d}$, upon electron or hole doping, the distortion is reduced compared the undoped system since $\mathrm{Mn}^{2+}$ and $\mathrm{Mn}^{4+}$ are not Jahn-Teller ions, causing $\mathrm{Mn}-\mathrm{O}$ bonds to become more symmetric. This enhances ferromagnetism of LMO due to the increased contribution from ferromagnetic exchange between quarter-filled $e_{\mathrm{g}}$ orbitals and the decreased contribution from antiferromagnetic super-exchange between half-filled $t_{2 \mathrm{~g}}$ orbitals ${ }^{39}$. The enhancement of ferromagnetism with electron or hole doping is consistent with experimental ${ }^{40,41}$ and theoretical ${ }^{42,43}$ results for the chemically doped LMO.

Discussion. Furthermore, it should be noted that the creation of the electron-doped ferromagnetic manganite was not obvious, being a challenging fundamental question full of controversy. Generally, the chemically doped $n$-type manganite always showed impurity phase and $p$-type behavior, and the conventional double or superexchange in electron-gated systems does not lead to ferromagnetism. Examples are $\mathrm{La}_{1-x} \mathrm{Ce}_{x} \mathrm{MnO}_{3}{ }^{23,44,45}, \mathrm{La}_{1}$ ${ }_{-x} \mathrm{Sb}_{x} \mathrm{MnO}_{3}{ }^{46}$, and $\mathrm{La}_{1-x} \mathrm{Te}_{x} \mathrm{MnO}_{3}{ }^{47}$ etc, which always show impurity phase and $p$-type behavior. In addition, the conventional double-exchange/super-exchange in electron-gated systems does not lead to ferromagnetism, we therefore believe that the ambipolar ferromagnetic in manganite is unexpected in a hard doping situation. In addition, since there is no report on the AHE in
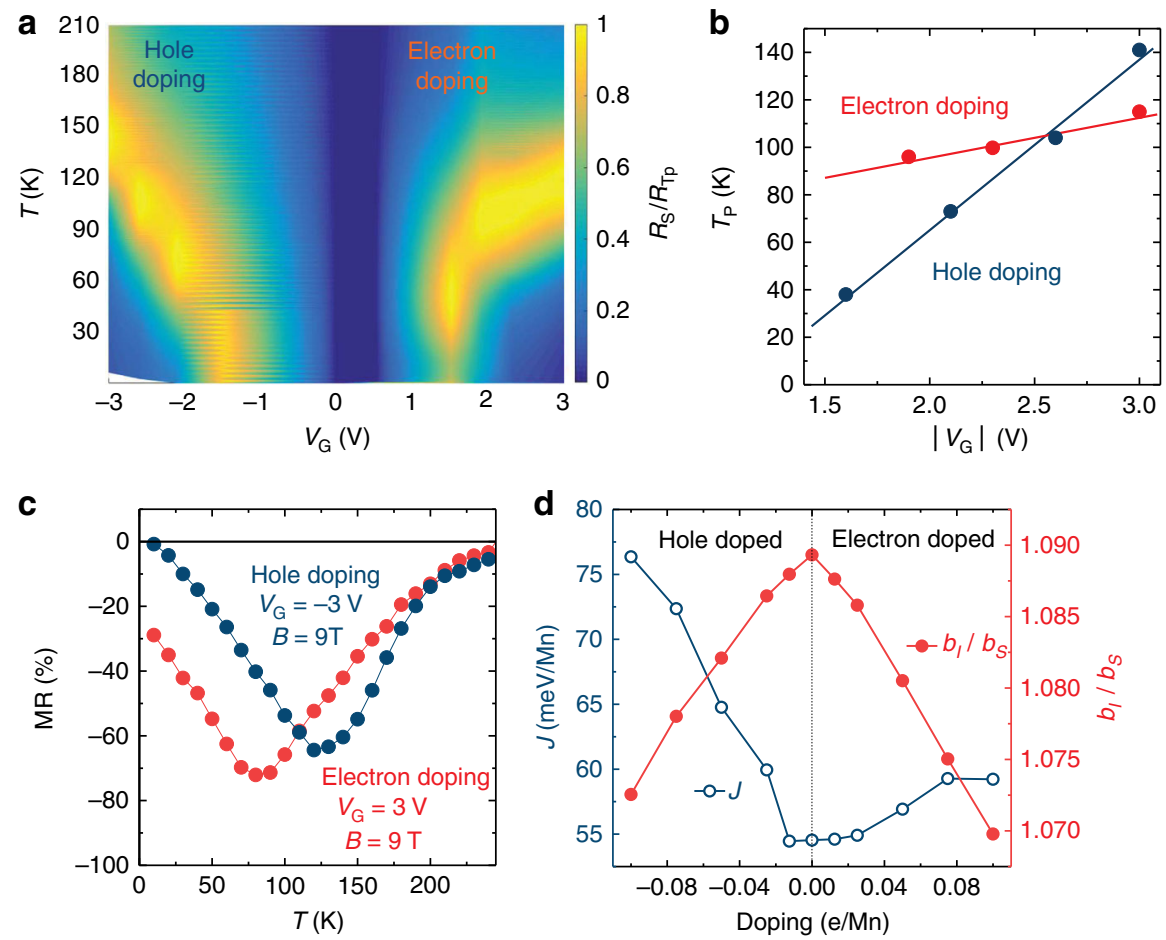

Fig. 4 Electron-hole asymmetry in electrostatically gated $\mathrm{LaMnO}_{3}$. a Contour plot of sheet resistance $\left(R_{\mathrm{S}}\right)$ as a function of gate voltage $\left(V_{\mathrm{G}}\right)$ and temperature. $\mathbf{b}$ Evolution of the resistivity peak $\left(T_{\mathrm{P}}\right)$ of $R_{\mathrm{S}}$ in the electron-doped and hole-doped regimes. $\mathbf{c}$ Asymmetric peak magnitude and temperature of the magnetoresistance $(\mathrm{MR})$, defined as $(R(H)-R(0)) / R(0))(\%)$, for an electron-doped or hole-doped $\mathrm{LaMnO}_{3}$ film. $\mathbf{d}$ First-principles calculations on the magnetism of $\mathrm{LaMnO}_{3}$ under bipolar gating. The exchange coupling parameter and $J$ (open blue dots) and the distortion parameter $b_{1} / b_{s}$ (filled red dots) are shown as a function of doping 
electron-doped manganites yet, our observation completes the electron-doped region of the phase diagram of manganite thin film.

In conclusion, we have induced ambipolar ferromagnetism with a Curie temperature greater than $100 \mathrm{~K}$ in ultrathin films of LMO by bipolar gating. We found that the electrostatic doping of this material with either electrons or holes strengthens the ferromagnetic order in this compound, with a pronounced electron-hole asymmetry seen in the insulator-to-metal transition as well as the CMR effect. The ambipolar ferromagnet is a missing link between spintronics and semiconductor physics. Electric control of the charge of spin-polarized carriers offers an opportunity for future bipolar magnetic technology in strongly correlated electron systems.

\section{Methods}

Device fabrication. Three uc-thick LMO films were grown on $\mathrm{TiO}_{2}$-terminated STO (001) at $750^{\circ} \mathrm{C}$ using pulsed laser deposition in an oxygen pressure of $10{ }^{2}$ mbar. The LMO growth is layer-by-layer and was monitored by in situ reflection high energy electron diffraction. After deposition, all samples were cooled down to room temperature in oxygen at the deposition pressure. The laser pulse $(248 \mathrm{~nm})$ energy density was $1.8 \mathrm{~J} \mathrm{~cm}^{-2}$ and the repetition rate was $1 \mathrm{~Hz}$. Subsequently, the film was patterned into a Hall bar using photolithography.

Electrical measurements. Carrier doping was realized by applying gate voltages $\left(V_{\mathrm{G}}\right)$ on the double-layer capacitor formed by the ionic liquid. The ionic liquid used in the experiment was a small droplet of [ $\mathrm{N}$-diethyl- $\mathrm{N}$-methyl- $\mathrm{N}$-(2-methoxyethyl) ammonium bis (trifluoromethyl sulphonyl) imide] covering both the conducting channel and the gate electrode. The leakage current was less than $1 \mathrm{nA}$ at a gate bias of $V_{\mathrm{G}}= \pm 3 \mathrm{~V}$. The electrical resistivity of the gated LMO films was measured using a Quantum Design Physical Property Measurement System (PPMS), a Keithley 2400 source measurement unit, and a Keithley 2182 nanovoltmeter from $300 \mathrm{~K}$ to $2 \mathrm{~K}$ in a magnetic field of 9 Tesla. Gate voltages were applied at $300 \mathrm{~K}$ between ionic liquid and LMO thin film to inject electrons or holes in LMO films, and the desired gate voltage was maintained throughout the whole transport measurement. Figure $4 \mathrm{a}$ is a re-plot of the data shown in Fig. 3a, b in MatLab using three standard plotting functions, namely Delaunay, trisurf, and shading. No manual averaging was done on the date and the horizontal stripes in Fig. $4 \mathrm{a}$ are generated due to the noise in resistance values in the raw data.

STEM measurements. High-resolution cross-section STEM-HAADF imaging and EELS were performed using the JEOL-ARM200F microscope equipped with ASCOR aberration corrector and cold-field emission gun and operated at $200 \mathrm{kV}$. The cross-section TEM sample was prepared by focused ion beam with $30 \mathrm{kV} \mathrm{Ga}$ ions, followed by a $2 \mathrm{kV}$ low voltage cleaning step. The HAADF images were acquired with a probe-forming aperture of $30 \mathrm{mrad}$ and collection angle of 68-280 mrad. All HAADF images were filtered by radial Wiener filters. EELS spectra were recorded using a Gatan Quantum ER spectrometer with a $0.25 \mathrm{eV} /$ channel energy dispersion.

DFT calculations. Theoretical modeling of the orthorhombic Pbnm LMO was performed using density functional theory, the projected augmented wave method, and PBEsol pseudopotentials, as implemented in the Vienna ab initio simulation package. Internal coordinates were fully relaxed, assuming an experimental lattice constant of LMO and using the force convergence limit of $1 \mathrm{meV} /$ atom. Correlation effects beyond generalized gradient approximation (GGA) were treated at a semi-empirical GGA $+U$ level within a rotationally invariant formalism with $U=$ $5 \mathrm{eV}$ for the Mn $3 d$-orbitals.

Data availability. The authors declare that all other relevant data supporting the findings of the study are available in this article and in its Supplementary Information file. Access to our raw data can be obtained from the corresponding author upon reasonable request.

Received: 3 October 2017 Accepted: 12 April 2018

Published online: 15 May 2018

\section{References}

1. Williams, J. R., DiCarlo, L. \& Marcus, C. M. Quantum Hall effect in a gatecontrolled p-n junction of graphene. Science 317, 638-641 (2014).
2. Li, L. et al. Black phosphorus field-effect transistors. Nat. Nanotech. $\mathbf{9}$, 372-377 (2014).

3. Meijer, E. J. et al. Solution-processed ambipolar organic field-effect transistors and inverters. Nat. Mater. 2, 678-682 (2003).

4. Ueno, K. et al. Electric-field-induced superconductivity in an insulator. Nat. Mater. 7, 855-858 (2008).

5. Goldman, A. M. Electrostatic gating of ultrathin films. Annu. Rev. Mater. Res 44, 45-63 (2014).

6. Salamon, M. B. \& Jaime, M. The physics of manganites: structure and transport. Rev. Mod. Phys. 73, 583-628 (2001).

7. Ye, J. T. et al. Liquid-gated interface superconductivity on an atomically flat film. Nat. Mater. 9, 125-128 (2010).

8. Ueno, K. et al. Discovery of superconductivity in $\mathrm{KTaO}_{3}$ by electrostatic carrier doping. Nat. Nanotech. 6, 408-412 (2011).

9. Bollinger, A. T. et al. Superconductor-insulator transition in $\mathrm{La}_{2-x} \mathrm{Sr}_{x} \mathrm{CuO}_{4}$ at the pair quantum resistance. Nature 472, 458-460 (2011).

10. Ye, J. T. et al. Superconducting Dome in a gate-tuned band insulator. Science 338, 1193-1196 (2012).

11. $\mathrm{Lu}, \mathrm{J}$. M. et al. Evidence for two-dimensional Ising superconductivity in gated $\mathrm{MoS}_{2}$. Science 350, 1353-1357 (2015).

12. Saito, Y., Kasahara, Y., Ye, J., Iwasa, Y. \& Nojima, T. Metallic ground state in an ion-gated two-dimensional superconductor. Science 350, 409-413 (2015).

13. Costanzo, D., Jo, S., Berger, H. \& Morpurgo, A. F. Gate-induced superconductivity in atomically thin $\mathrm{MoS}_{2}$ crystals. Nat. Nanotech. 11, 339-344 (2015).

14. Shiogai, J., Ito, Y., Mitsuhashi, T., Nojima, T. \& Tsukazaki, A. Electric-fieldinduced superconductivity in electrochemically-etched ultrathin FeSe films on $\mathrm{SrTiO}_{3}$ and MgO. Nat. Phys. 12, 42-46 (2016).

15. Li, L. J. et al. Controlling many-body states by the electric-field effect in a twodimensional material. Nature 529, 185-189 (2015).

16. Nakano, M. et al. Collective bulk carrier delocalization driven by electrostatic surface charge accumulation. Nature 487, 459-462 (2012).

17. Jeong, J. et al. Suppression of metal-insulator transition in $\mathrm{VO}_{2}$ by electric field-induced oxygen vacancy formation. Science 339, 1402-1405 (2013).

18. Dhoot, A. S., Israel, C., Moya, X., Mathur, N. D. \& Friend, R. H. Large electric field effect in electrolyte-gated manganites. Phys. Rev. Lett. 102, 136402 (2009).

19. Hatano, T. et al. Gate control of electronic phases in a quarter-filled manganite. Sci. Rep. 3, 2904 (2013).

20. Lourembam, J., Wu, J., Ding, J., Lin, W. \& Wu, T. Electric field tuning of phase separation in manganite thin films. Phys. Rev. B 89, 014425 (2014).

21. Cui, B. et al. Reversible ferromagnetic phase transition in electrode- gated manganites. Adv. Funct. Mater. 24, 7233-7240 (2014).

22. Coey, J. M. D., Viret, M. \& Molnar, Svon Mixed-valence manganites. Adv. Phys. 48, 167-293 (1999).

23. Werner, R. et al. Transport, magnetic, and structural properties of $\mathrm{La}_{0.7} \mathrm{Ce}_{0.3} \mathrm{MnO}_{3}$ thin films: evidence for hole-doping. Phys. Rev. B 79, 054416 (2009).

24. Renshaw Wang, X. et al. Imaging and control of ferromagnetism in $\mathrm{LaMnO}_{3} /$ $\mathrm{SrTiO}_{3}$ heterostructures. Science 349, 716-719 (2015).

25. Peng, J. J. et al. Restoring the magnetism of ultrathin $\mathrm{LaMnO}_{3}$ films by surface symmetry engineering. Phys. Rev. B 94, 214404 (2016).

26. Song, C., Cui, B., Li, F., Zhou, X. \& Pan, F. Recent progress in voltage control of magnetism: materials, mechanisms, and performance. Prog. Mater. Sci. 87, 33-82 (2017).

27. Wang, L. M. Anomalous Hall effect in $\mathrm{La}_{0.7} \mathrm{Sr}_{0.3} \mathrm{MnO}_{3} / \mathrm{SrTiO}_{3}$ superlattices: hopping transport and a probe of dimensionality. Phys. Rev. Lett. 96, 077203 (2006).

28. Nagaosa, N., Sinova, J., Onoda, S., MacDonald, A. H. \& Ong, N. P. Anomalous Hall effect. Rev. Mod. Phys. 82, 1539-1592 (2010).

29. Malfait, M. et al. Sign inversion of the high-field Hall slope in epitaxial $\mathrm{La}_{0.5} \mathrm{Ca}_{0.5} \mathrm{MnO}_{3}$ thin films. Phys. Rev. B 68, 132410 (2003).

30. Chun, S. H., Salamon, M. B. \& Han, P. D. Hall effect of $\mathrm{La}_{2 / 3}(\mathrm{Ca}, \mathrm{Pb})_{1 / 3} \mathrm{MnO}_{3}$ single crystals near the critical temperature. Phys. Rev. B 59, 11155-11158 (1999).

31. Lyanda-Geller, Y. et al. Charge transport in manganites: hopping conduction, the anomalous Hall effect, and universal scaling. Phys. Rev. B 63, 184426 (2001).

32. Nair, S. et al. Hall effect in heavy fermion metals. Adv. Phys. 61, 583-664 (2012).

33. Majumdar, P., Simon, S. H. \& Sengupta, A. M. Hall effect in the perovskite manganites. Phys. Rev. B 59, 4746-4751 (1999).

34. Zhang, Y., Ye, J., Matsuhashi, Y. \& Iwasa, Y. Ambipolar $\mathrm{MoS}_{2}$ thin flake transistors. Nano. Lett. 12, 1136-1140 (2012).

35. Saito, Y. \& Iwasa, Y. Ambipolar insulator-to-metal transition in black phosphorus by ionic-liquid gating. ACS Nano 9, 3192-3198 (2015).

36. Millis, A. J. Lattice effects in magnetoresistive manganese perovskites. Nature 392, 147-150 (1998). 
37. Coey, J. M. D., Venkatesan, M. \& Stamenov, P. Surface magnetism of strontium titanate. J. Phys. Condens. Matter 28, 485001 (2016).

38. Yamauchi, K., Freimuth, F., Blügel, S. \& Picozzi, S. Magnetically induced ferroelectricity in orthorhombic manganites: Microscopic origin and chemical trends. Phys. Rev. B 78, 014403 (2008).

39. Solovyev, I., Hamada, N. \& Terakura, K. Crucial role of the lattice distortion in the magnetism of $\mathrm{LaMnO}_{3}$. Phys. Rev. Lett. 76, 4825-4828 (1996).

40. Raychaudhuri, P., Mitra, C., Mann, P. D. A. \& Wirth, S. Phase diagram and Hall effect of the electron doped manganite $\mathrm{La}_{1-x} \mathrm{Ce}_{x} \mathrm{MnO}_{3}$. J. Appl. Phys. 93, 8328-8330 (2003).

41. Fujishiro, H., Fukase, T. \& Ikebe, M. Anomalous lattice softening at $X=0.19$ and 0.82 in $\mathrm{La}_{1-x} \mathrm{Ca}_{x} \mathrm{MnO}_{3}$. J. Phys. Soc. Jpn. 70, 628-631 (2001).

42. Schlottmann, P. Correlated electrons in manganites. Phys. Rev. B 62, 439-446 (2000).

43. Schlottmann, P. Phase diagram and spin-canting effects in electron-doped $\mathrm{LaMnO}_{3}$. Phys. Rev. B 77, 104446 (2008).

44. Wang, D. J. et al. Hall effect in $\mathrm{La}_{0.7} \mathrm{Ce}_{0.3} \mathrm{MnO}_{3+\delta}$ films with variable oxygen content. Phys. Rev. B 73, 144403 (2006).

45. Mandal, P. \& Das, S. Transport properties of Ce-doped $\mathrm{RMnO}_{3}(\mathrm{R}=\mathrm{La}, \mathrm{Pr}$, and Nd) manganites. Phys. Rev. B 56, 15073 (1997).

46. Wang, D., Wang, M., Wang, R. \& Li, Y. Hall effect of quinquevalent ion-doped $\mathrm{La}_{0.9} \mathrm{Sb}_{0.1} \mathrm{MnO}_{3}$ film. J. Rare Earths 31, 257-261 (2013).

47. Tan, G. T. et al. Colossal magnetoresistance behavior and ESR studies of $\mathrm{La}_{1-X} \mathrm{Te}_{X} \mathrm{MnO}_{3}(0.04<X<0.2)$. Phys. Rev. B 68, 014426 (2003).

\section{Acknowledgements}

L.M.Z., W.M.L., and W.W.C. acknowledges supports from the National Science Foundation of China (No. 51602080 and 51572055), the National Key Basic Research Program of China (No. 2103CB632900) and PIRS of HIT (No. 201625). X.R.W. acknowledges supports from the Nanyang Assistant Professorship grant from Nanyang Technological University and Academic Research Fund Tier 1 (RG108/17S) from Singapore Ministry of Education. C.J.L. acknowledges the financial support from the Lee Kuan Yew Postdoctoral Fellowship through the Singapore Ministry of Education Academic Research Fund Tier 1 (R-284-000-158-114). J.M.D.C. acknowledges supports from China 1000 Foreign Talents Program and from Science Foundation Ireland, Grant 16/IA/4534. Z.H. C. acknowledges support from the National Science Foundation under grant DMR1608938. L.W.M. acknowledges support from the Gordon and Betty Moore Foundation's EPiQS Initiative, under grant GBMF5307. The research at the University of NebraskaLincoln is supported by the National Science Foundation through the Nebraska Materials Research Science and Engineering Center (NSF Grant No. DMR-1420645). Z. Q.L. acknowledges support from the National Science Foundation of China (NSFC Grant No. 11704018). The electron microscopy performed at ORNL (S.Z.Y. and M.F.C.) was supported by the U.S. Department of Energy's, Office of Science, Basic Energy Sciences,
Materials Sciences and Engineering Division. The electron microscopy (S.J.P., M.S.L., and C.J.L.) is supported by the National University of Singapore. This research used resources of the National Energy Research Scientific Computing Center, a DOE Office of Science User Facility supported by the Office of Science of the U.S. Department of Energy under Contract No. DE-AC02-05CH11231. This work used the Extreme Science and Engineering Discovery Environment (XSEDE), which is supported by National Science Foundation grant number ACI-1053575 and Grant No DMR160118.

\section{Author contributions}

X.R.W., W.M.L., and J.M.D.C. planned and supervised the research. L.M.Z. and W.M.L. performed sample fabrication and measurements. C.J.L., M.S.L., and S.J.P. performed STEM measurements. T.R.P. and E.Y.T. performed theoretical modeling. All authors contributed to the discussion and interpretation of the results. X.R.W., W. M. L., and J.M. D.C. wrote the manuscript with help from all other authors.

\section{Additional information}

Supplementary Information accompanies this paper at https://doi.org/10.1038/s41467 018-04233-5.

Competing interests: The authors declare no competing interests.

Reprints and permission information is available online at http://npg.nature.com/ reprintsandpermissions/

Publisher's note: Springer Nature remains neutral with regard to jurisdictional claims in published maps and institutional affiliations.

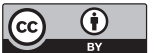

Open Access This article is licensed under a Creative Commons Attribution 4.0 International License, which permits use, sharing, adaptation, distribution and reproduction in any medium or format, as long as you give appropriate credit to the original author(s) and the source, provide a link to the Creative Commons license, and indicate if changes were made. The images or other third party material in this article are included in the article's Creative Commons license, unless indicated otherwise in a credit line to the material. If material is not included in the article's Creative Commons license and your intended use is not permitted by statutory regulation or exceeds the permitted use, you will need to obtain permission directly from the copyright holder. To view a copy of this license, visit http://creativecommons.org/ licenses/by/4.0/.

(C) The Author(s) 2018 